\title{
Dynamics in inhomogeneous liquids and glasses via the test particle limit
}

\author{
Andrew J. Archer, ${ }^{1,2}$ Paul Hopkins, ${ }^{2}$ and Matthias Schmidt ${ }^{2,3}$ \\ ${ }^{1}$ Department of Mathematical Sciences, Loughborough University, Loughborough, Leicestershire LE11 3TU, United Kingdom \\ ${ }^{2}$ H.H. Wills Physics Laboratory, University of Bristol, Tyndall Avenue, Bristol BS8 1TL, United Kingdom \\ ${ }^{3}$ Institut für Theoretische Physik II, Heinrich-Heine-Universität Düsseldorf, Universitätsstraße 1, D-40225 Düsseldorf, Germany
}

(Received 26 September 2006; revised manuscript received 27 February 2007; published 23 April 2007)

\begin{abstract}
We show that one may view the self-part and the distinct-part of the van Hove dynamic correlation function of a simple fluid as the one-body density distributions of a binary mixture that evolve in time according to dynamical density functional theory. For a test case of soft-core Brownian particles the theory yields results for the van Hove function that agree quantitatively with those of our Brownian dynamics computer simulations. At sufficiently high densities the free energy landscape underlying the dynamics exhibits a barrier as a function of the mean particle displacement, shedding new light on the nature of glass formation. For hard spheres confined between parallel planar walls the barrier height oscillates in phase with the local density, implying that the mobility is maximal between layers, which should be experimentally observable in confined colloidal dispersions.
\end{abstract}

DOI: 10.1103/PhysRevE.75.040501

PACS number(s): 64.70.Pf, 61.20.Lc, 82.70.Dd

The van Hove function $G(r, t)$ for the probability of finding a particle at time $t$ at a distance $r$ from the origin, given that there was a particle at the origin at time $t=0$, characterizes dynamical phenomena in condensed matter on a nanoscopic scale $[1,2]$. Recent measurements of $G(r, t)$ using confocal microscopy were aimed at investigating, e.g., dynamical heterogeneities in colloidal hard-sphere suspensions [3] or the devitrification of colloidal glasses upon adding nonadsorbing polymers [4]. The Fourier transform of $G(r, t)$, the intermediate scattering function, can be measured with inelastic scattering techniques [2].

There is immense interest in deriving theoretical approaches for investigating the microscopic dynamics in dense inhomogeneous liquids [5], relevant to studying, e.g., the glass transition of liquids adsorbed in nanoporous materials [6], gravity-induced aging in glasses of colloidal hard spheres [7], and gaining understanding of molecular diffusion inside of technologically relevant materials, such as zeolites [8]. In the static case modern density functional theory (DFT) provides a powerful means for investigating the significant effects on the phase behavior and structural correlations that spatial confinement and external fields may induce $[2,9]$. DFT operates on the level of the one-body density distribution $\rho(\mathbf{r})$, where $\mathbf{r}$ is the space coordinate. For a range of situations the time evolution of the density profile, $\rho(\mathbf{r}, t)$, as induced by a time-dependent external potential $V_{\text {ext }}(\mathbf{r}, t)$, has been shown to be well described by dynamical density functional theory (DDFT) [10,11]; see, e.g., the successful applications in Refs. $[10,12,13]$. However, currently no similar framework exists for calculating dynamical two-body correlation functions, as required to address problems such as those mentioned above.

In the static case a close relationship between $\rho(\mathbf{r})$ and the pair distribution function $g(r)$, describing the probability of finding a pair of particles separated by a distance $r$, is established by Percus' famous test particle limit [14]: $\rho g(r)$ is the one-body density distribution of a fluid exposed to the influence of an external potential $V_{\text {ext }}(r)$, which represents a "test particle" fixed at the origin, given by $V_{\text {ext }}(r)=V(r)$, where
$V(r)$ is the interparticle pair potential and $\rho$ is the bulk density.

In this Rapid Communication we generalize the test particle limit to dynamical correlation functions, allowing us to use DDFT to calculate the van Hove function in bulk and in inhomogeneous systems. We test this approach by comparing results to those from our Brownian dynamics computer simulations for a simple Gaussian core model (GCM) for a macromolecular solution in bulk and indeed find very good agreement. For hard spheres we estimate the glass transition to be at bulk densities $\rho \sigma^{3} \simeq 0.9$, where $\sigma$ is the hard-sphere diameter. Upon confining the hard-sphere system between parallel hard walls, we find the local mobility to be maximal where the local density is minimal. Our approach allows for inspection of a well-defined underlying free-energy landscape, which requires solving a corresponding equilibrium DFT rather than the full DDFT, easing numerical burdens.

Consider a fluid of $N$ particles that interact via a pair potential $V(r)$. The system may be viewed as a binary mixture of species $s$ and $d$, in which two of the pair potentials $V_{i j}(r)$ between particles of species $i, j=s, d$ are equal, $V_{d d}(r)=V_{s d}(r)=V(r)$ and $V_{s s}(r)=0$. Furthermore, species $s$ consists of only a single (test) particle, located at position $\mathbf{r}_{0}$ at $t=0$, while species $d$ consists of the remaining $N-1$ particles of the system. Our aim is to relate the one-body density distributions of this mixture, $\rho_{s}(\mathbf{r}, t)$ and $\rho_{d}(\mathbf{r}, t)$, to the selfpart and distinct-part of the van Hove function of the pure fluid, $G_{s}\left(\mathbf{r}_{0}, \mathbf{r}, t\right)$ and $G_{d}\left(\mathbf{r}_{0}, \mathbf{r}, t\right)$, respectively. [Recall that $G(r, t)=G_{s}(r, t)+G_{d}(r, t)$.] We must choose suitable initial conditions for $\rho_{s}(\mathbf{r}, t=0)$ and $\rho_{d}(\mathbf{r}, t=0)$. In the case of a bulk system let the one-body density distribution of species $s$ at time $t=0$ be $\rho_{s}(\mathbf{r}, t=0)=\delta(\mathbf{r})$, where $\delta(\cdot)$ is the Dirac delta function and we have chosen the coordinate system such that $\mathbf{r}_{0}=\mathbf{0}$. Species $d$ is initially at equilibrium-i.e., $\rho_{d}(\mathbf{r}, t=0)$ $=\rho g(r)$. For subsequent times $t>0$, we make the identification $G_{s}(r, t)=\rho_{s}(r, t)$ and $G_{d}(r, t)=\rho_{d}(r, t)$.

There are two ways to proceed. One is to carry out computer simulations on the level of particle coordinates (detailed below). The alternative is to use a theory that operates 
on the one-body level, and DDFT is an obvious choice. Generally there are only formal results for the exact equations for the time evolution and hence one must resort to approximations $[10,11,15,16]$, such as the theory of Marconi and Tarazona [10] for Brownian dynamics:

$$
\frac{\partial \rho_{i}}{\partial t}=\Gamma \nabla \cdot\left(\rho_{i} \nabla \frac{\delta F\left[\rho_{s}, \rho_{d}\right]}{\delta \rho_{i}}\right), \quad i=s, d,
$$

where $\Gamma$ is a friction coefficient characterizing the drag of the solvent on the particles and $F\left[\rho_{s}, \rho_{d}\right]$ is taken to be the equilibrium Helmholtz free-energy functional:

$$
\begin{aligned}
F\left[\rho_{s}, \rho_{d}\right]= & k_{B} T \sum_{i=s, d} \int d \mathbf{r} \rho_{i}(\mathbf{r})\left\{\ln \left[\rho_{i}(\mathbf{r}) \Lambda^{3}\right]-1\right\}+F_{\mathrm{ex}}\left[\rho_{s}, \rho_{d}\right] \\
& +\int d \mathbf{r} V_{\mathrm{ext}}(\mathbf{r})\left[\rho_{s}(\mathbf{r})+\rho_{d}(\mathbf{r})\right],
\end{aligned}
$$

where the first term on the right-hand side is the Helmholtz free energy functional of the (binary) ideal gas, $\Lambda$ is the (irrelevant) thermal wavelength, $T$ is the temperature, $k_{B}$ is the Boltzmann constant, and $F_{\text {ex }}\left[\rho_{s}, \rho_{d}\right]$ is the excess contribution to the Helmholtz free energy due to interactions between the particles [18]. For pioneering work that leads to Eqs. (1) and (2) see [17].

As a test case we consider the GCM, which describes the soft interactions of polymer coils, star polymers, or dendrimers in solution [19] and is defined by $V(r)=\epsilon \exp \left(-r^{2} / R^{2}\right)$; $R$ is the radius of the particles and $\epsilon$ is the energy cost for complete overlap of a pair of particles. We use a simple mean-field approximation $F_{\text {ex }}\left[\rho_{s}, \rho_{d}\right]$ $=\frac{1}{2} \sum_{i, j=s, d} \int d \mathbf{r} d \mathbf{r}^{\prime} \rho_{i}(\mathbf{r}) V_{i j}\left(\left|\mathbf{r}-\mathbf{r}^{\prime}\right|\right) \rho_{j}\left(\mathbf{r}^{\prime}\right)$, which has been shown to be reliable for this model [12], and integrate Eq. (1) numerically with the given initial conditions forward in time. In Fig. 1 results are displayed for both parts of the van Hove function at four different times for a typical state point. We also display results from Brownian dynamics (BD) simulations of 300 particles, with a time step of $10^{-3} \tau_{B}$, where $\tau_{B}$ $=R^{2} /\left(\Gamma k_{B} T\right)$. The very good agreement between the DDFT and BD simulation results attests to the validity of our scheme. Nevertheless, a remark about the more general applicability of the DDFT in Eq. (1) is in order. The relevant dynamical variable is the root-mean-square particle displacement $w(t)$, defined as the width of $G_{s}(r, t)$ [and hence of $\left.\rho_{s}(r, t)\right]$ via

$$
[w(t)]^{2}=4 \pi \int_{0}^{\infty} d r r^{4} G_{s}(r, t) .
$$

For $t \rightarrow \infty, w(t)^{2}=6 D_{L} t$, where $D_{L}$ is the long-time selfdiffusion coefficient [2]. However, for not too low temperatures and $t \rightarrow \infty$, Eq. (1) predicts that $w(t)^{2}=6 D_{S} t$, where $D_{S}=k_{B} T / \Gamma$ is the short-time self-diffusion coefficient, and for the GCM, $D_{L} \simeq D_{S}[16]$. However, for particles with a hard core $D_{L}<D_{S}$ and the DDFT in Eq. (1) is not reliable for $t \gg \tau_{B}$. To circumvent this problem one could use a DDFT such as that of Ref. [16], which guarantees the correct longtime behavior - see also the approach of Ref. [20].

Here we choose a different direction and investigate
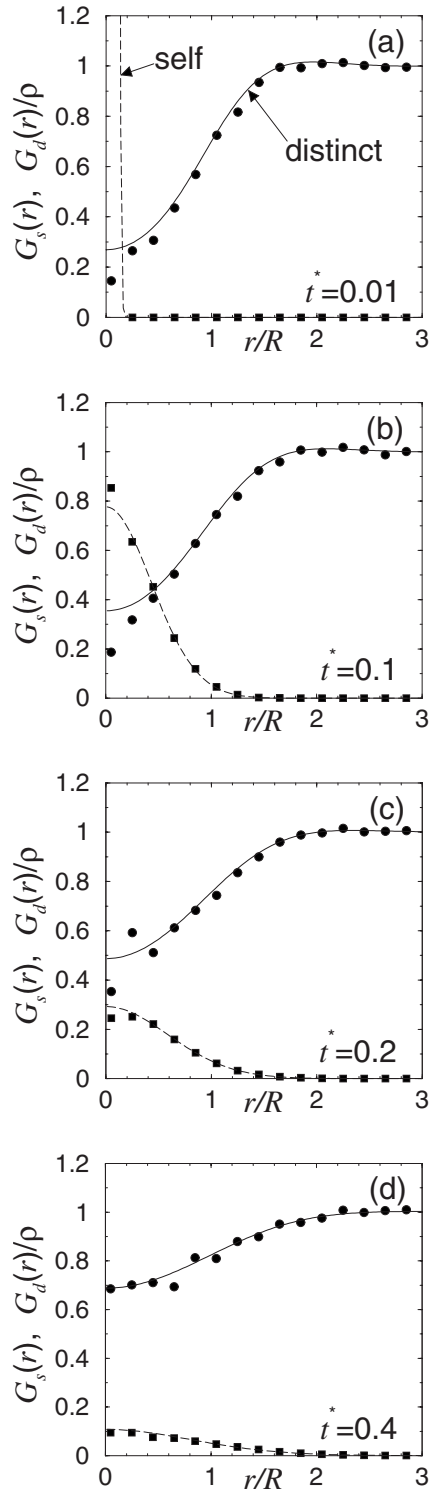

FIG. 1. The self-part $G_{s}(r, t)$ (dashed lines, squares) and scaled distinct-part $G_{d}(r, t) / \rho$ (solid lines, circles) of the van Hove correlation function for the GCM fluid with bulk density $\rho R^{3}=0.2263$ and $k_{B} T / \epsilon=0.5$, at times $t^{*} \equiv t / \tau_{B}=0.01,0.1,0.2,0.4$ [(a),(b),(c),(d), respectively], as a function of the (scaled) distance $r / R$. Shown are results from DDFT (lines) and BD simulations (symbols).

the free-energy landscape underlying Eq. (1) in more detail. We consider one of the fundamental models of liquid-state physics: namely, a one-component system of hard spheres. This is described by the Ramakrishnan-Yussouff (RY) approximation $\quad[2,21] \quad F_{\mathrm{ex}}\left[\rho_{s}, \rho_{d}\right]=V f_{\mathrm{ex}}(\rho)+\Sigma_{i=s, d} f_{\mathrm{ex}}^{\prime}(\rho)$ $\times \int d \mathbf{r} \Delta \rho_{i}(\mathbf{r})-\frac{1}{2} \sum_{i, j=s, d} \int d \mathbf{r} d \mathbf{r}^{\prime} \Delta \rho_{i}(\mathbf{r}) c_{i j}\left(\left|\mathbf{r}-\mathbf{r}^{\prime}\right|\right) \Delta \rho_{j}\left(\mathbf{r}^{\prime}\right)$, where $f_{\text {ex }}(\rho)$ is the bulk excess free energy per volume $V$, $f_{\mathrm{ex}}^{\prime}(\rho)=\partial f_{\mathrm{ex}}(\rho) / \partial \rho, \Delta \rho_{i}(r)=\rho_{i}(r)-\rho_{i}$, with $\rho_{d}=\rho, \rho_{s}=0$, and the Percus-Yevick approximation [2] for the pair direct correlation function $c_{i j}(r)$; we set $c_{s s}(r)=0$ to model the absence of test particle self-interactions. Chosen here for its simplicity, this approximation for $F_{\text {ex }}\left[\rho_{s}, \rho_{d}\right]$ is crude, but sufficient to explore qualitative behavior. We use equilibrium DFT to elucidate a pathway that closely resembles that of the full 


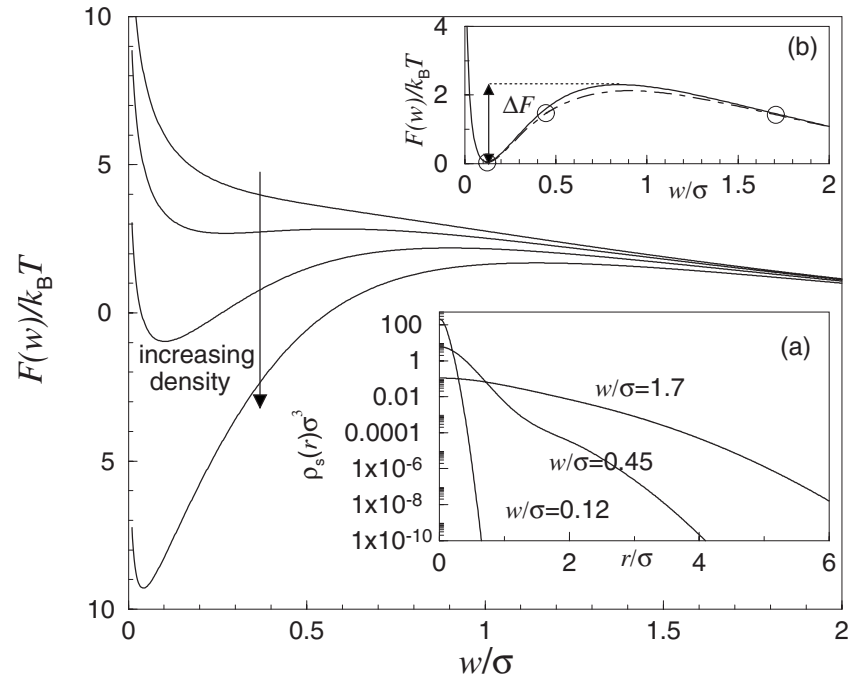

FIG. 2. The scaled free energy $F / k_{B} T$ as a function of the rootmean-square particle displacement $w$ [see Eq. (3)], calculated with the RY functional for hard spheres at densities $\rho \sigma^{3}=0.6,0.7,0.8$, and 0.9 (as indicated by the arrow) and shifted such that $F(w=3)$ $=0$. (a) Self-part of the van Hove function on a logarithmic scale as a function of $r / \sigma$ for $\rho \sigma^{3}=0.78$ and three typical widths $w$. Note the pronounced non-Gaussian character for $w / \sigma=0.45$. (b) Comparison of results for $F / k_{B} T$ as a function of $w / \sigma$ from free minimization (dot-dashed line) and a Gaussian parametrization for $G_{s}$ (solid line) for $\rho \sigma^{3}=0.78$. Also shown is the free-energy barrier $\Delta F$ between minimum and maximum (arrow) and the values of $w / \sigma$ (circles) for which results are shown in (a).

DDFT by minimizing the free-energy functional [9]-i.e., solve $\delta F / \delta \rho_{i}(\mathbf{r})=\mu_{i}$ for $i=s, d$ where $\mu_{i}$ are Lagrange multipliers to fulfill $\int d \mathbf{r} \rho_{s}(\mathbf{r})=1$ and $\int d \mathbf{r}\left[\rho_{d}(\mathbf{r})-\rho\right]$ $=\rho \int d \mathbf{r}[g(r)-1]=$ const. As a constraint we control $w$ via an associated Lagrange multiplier $\lambda$, which is formally equivalent to treating an auxiliary external field acting on component $s$ with the simple harmonic form $V_{\text {ext }}^{(s)}(r)=\lambda r^{2} / \sigma^{2}$.

By calculating $F$ as a function of $\lambda$ (and hence $w$ ) for a given state point, we may determine the free-energy landscape that governs the time evolution of $G(r, t)$. We find that at low densities $F(w)$ decreases monotonically with $w$, but as $\rho$ is increased above a threshold, $\rho>\rho^{*}, F(w)$ becomes nonmonotonic and develops a barrier; the barrier height $\Delta F$-i.e., the difference between the minimum and maximum-grows upon increasing $\rho$; see Fig. 2. While for both small and large values of $w, \rho_{s}(r ; \lambda)$ is nearly Gaussian, for intermediate values, particularly when $\rho \gtrsim \rho^{*}, \rho_{s}(r ; \lambda)$ has pronounced non-Gaussian characteristics; Fig. 2(a) shows results for $\rho_{s}(r)$ for three typical values of $w$. For states that correspond to barrier crossing $\rho_{s}(r)$ exhibits a pronounced shoulder, taken as a signature of dynamical heterogeneity $[3,22]$. However, assuming the Gaussian form for all values of $w$, as in the main plot of Fig. 2, does not affect $F(w)$ strongly and only slightly overestimates $\Delta F$ [see Fig. 2(b) for a comparison of results from free minimization and from the Gaussian parametrization]. The emergence of a barrier in $F(w)$ leads to a trapping in the DDFT for $\rho>\rho^{*}$ whereby, as $t \rightarrow \infty, w(t) \rightarrow w_{0}<\infty$, suggesting that the system is nonergodic. However, in reality, as long as the barrier is suffi-
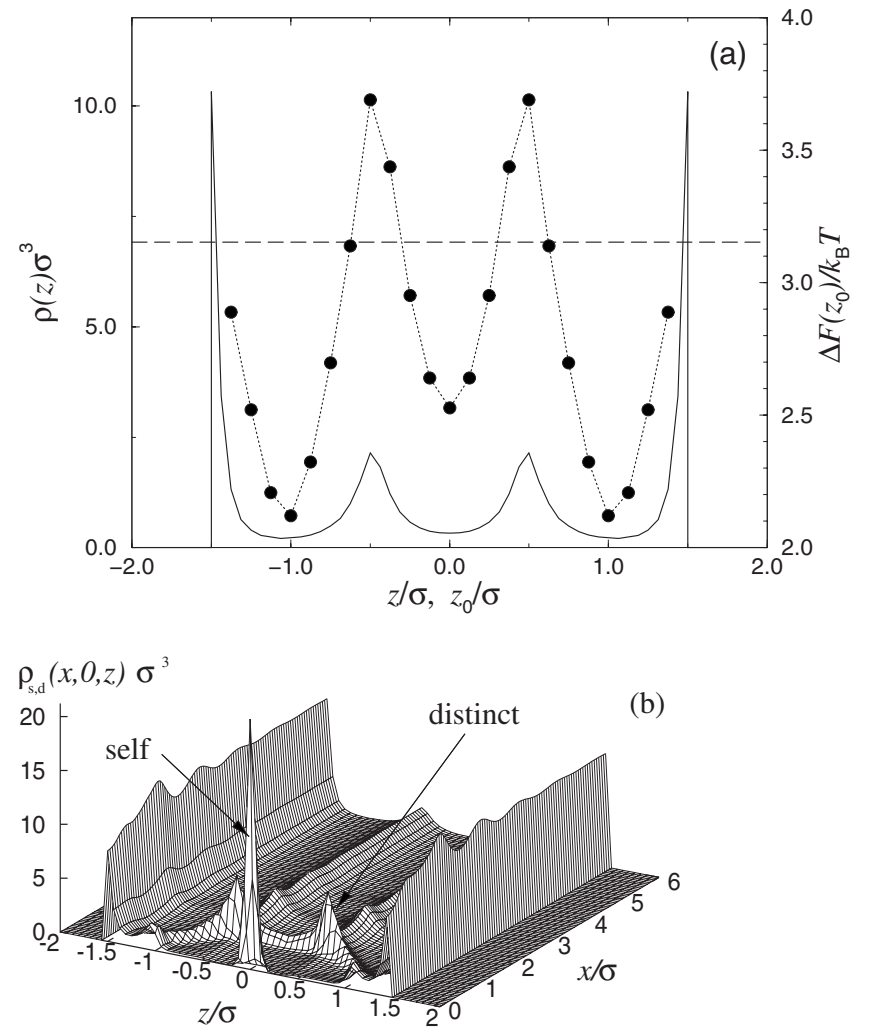

FIG. 3. Correlation functions for hard spheres confined between parallel plates of separation distance $H=4 \sigma$. (a) Density profile $\rho(z)$ (solid line) as a function of $z / \sigma$ and free-energy barrier $\Delta F\left(z_{0}\right)$ (symbols; the dotted line is to guide the eye). Also shown is the corresponding bulk value of $\Delta F$ (dashed line). (b) The (scaled) self-part $\rho_{s}(\mathbf{r}) \sigma^{3} / 25$ and distinct-part $\rho_{d}(\mathbf{r})$ of the van Hove function as a function of the scaled coordinates perpendicular and parallel to the wall, $z / \sigma$ and $x / \sigma$, respectively, evaluated at the minimum of the free-energy landscape for bulk density $\rho \sigma^{3}=0.8$.

ciently small, it can eventually be overcome. Our expression for $F_{\mathrm{ex}}$ is approximate and neglects some contributions to the free energy due to fluctuations $[9,23]$, so the particular DDFT in Eq. (1) does not describe the barrier crossing; one could include a stochastic noise term [20] to take this into account.

We may estimate the glass transition density of hard spheres by considering the time it takes to cross the barrier, $\tau=\tau_{0} e^{\Delta F / k_{B} T}$, where $\tau_{0}$ is a Brownian time scale $[20,24]$; in colloidal systems such as that of Ref. [3], $\tau_{0}$ is of the order of a few seconds. Our theory predicts a very sharp increase of $\tau$ with density; i.e., for $\rho \sigma^{3}=0.85$, we find $\Delta F=6.25 k_{B} T$ and $\tau=518 \tau_{0}$; for $\rho \sigma^{3}=0.89$, we obtain $\Delta F=9.87 k_{B} T$ and already $\tau=19300 \tau_{0}$. In the first case, the positions of the colloids would not be completely decorrelated from their initial positions after several minutes, and in the second case the system would certainly behave as a glass as $\tau$ is of the order of several hours. Hence one would expect the glass transition to occur around $\rho_{g} \sigma^{3} \simeq 0.9$, which is somewhat below the result $\rho_{g} \sigma^{3} \simeq 1.1$ found experimentally for colloidal hard spheres [25]. We attribute this underestimation to our choice of a simple (RY) free-energy functional; use of a more reliable functional [26] may improve results. 
Our approach shares some features in common with a number of other theories: (i) In the successful "nonclassical theory of nucleation" DFT is used to calculate the freeenergy barrier to nucleating a liquid droplet in the oversaturated gas [28]. (ii) In recent work, Schweizer and Saltzman $[20,24]$ construct a theory for the free energy of a single particle in the cage of its neighbors. However, we calculate the free energy of the entire system, including contributions from $\rho_{d}(r)$ and $\rho_{s}(r)$, whereas their theory is for the free energy of the test particle only. (iii) In Wolynes and coworkers' random first-order transition theory of glasses [27] DFT is used to calculate the free energy as a function of an appropriate order parameter. One key feature that discriminates our theory from the latter two approaches is that our approach is genuinely suited for studying inhomogeneous systems, as we demonstrate in the following.

We have investigated how the dynamics of hard spheres is affected by confinement between two planar parallel hard walls separated by a distance $H$, described by an external potential $V_{\text {ext }}(z)$ that vanishes for $-H / 2<z<H / 2$ and is infinite otherwise; $z$ is the coordinate perpendicular to the walls. The presence of the walls causes the one-body density $\rho(z)$ to be oscillatory with maximal value at contact with the walls; see Fig. 3(a) for results for a system with $H=4 \sigma$ in chemical equilibrium with a bulk of density $\rho \sigma^{3}=0.8$. Due to symmetry, the van Hove function depends on the initial position $z_{0}$ of the test particle, but not on its initial lateral co- ordinates, taken as $x_{0}=y_{0}=0$. We have used equilibrium DFT to determine $\rho_{d}(\mathbf{r})$, assuming $\rho_{s}(\mathbf{r})$ to be a Gaussian (recall that in bulk this assumption made little difference to the value of $F$ ). As an example we show in Fig. 3(b) results for $\rho_{s}(\mathbf{r})$ and $\rho_{d}(\mathbf{r})$ for the case where the test particle was initially in the midplane, $z_{0}=0$. We choose conditions (see below) where $\rho_{s}(\mathbf{r})$ is still peaked around $\mathbf{r}=0 ; \rho_{d}$ exhibits structure due to packing effects caused by the walls, as well as by packing around the test particle. Quite striking is the appearance of the hexagonal shape of the first coordination shell; for large planar distances $\rho_{d}(z, x \rightarrow \infty, y=0)=\rho(z)$. Similar to the bulk case we find a free-energy barrier $\Delta F$ that now depends on $z_{0}$ [the profiles in Fig. 3(b) are shown at the minimum of $F$ ]. Figure 3(a) shows strong oscillations of $\Delta F\left(z_{0}\right)$ around its value in bulk; the oscillations are in phase with the oscillations of $\rho(z)$. Since the barrier hopping time $\tau \sim e^{\Delta F / k_{B} T}$, the particles in the layers [i.e., where $\rho\left(z_{0}\right)$ is large] are less mobile than those between the layers. For the case in Fig. 3(b) the particles at $z= \pm 0.5 \sigma$ have a mobility $(\sim 1 / \tau)$ that is about $60 \%$ of the bulk value. This prediction can be tested by experiment with confined colloidal hard spheres or with computer simulations.

We thank R. Evans, M. Fuchs, J. M. Brader, and R. van Roij for valuable discussions. A.J.A. and P.H. are grateful for the support of EPSRC, and M.S. thanks the DFG for support through the SFB TR6/D3.
[1] L. van Hove, Phys. Rev. 95, 249 (1954).

[2] J.-P. Hansen and I. R. McDonald, Theory of Simple Liquids, 3rd ed. (Academic, London, 2006).

[3] W. K. Kegel and A. van Blaaderen, Science 287, 290 (2000).

[4] N. B. Simeonova et al., Phys. Rev. E 73, 041401 (2006).

[5] See, e.g., V. Krakoviack, Phys. Rev. Lett. 94, 065703 (2005); G. Biroli, J.-P. Bouchaud, K. Miyazaki, and D. R. Reichman, ibid. 97, 195701 (2006).

[6] M. Alcoutlabi and G. B. McKenna, J. Phys.: Condens. Matter 17, R461 (2005); C. Alba-Simionesco et al., ibid. 18, R15 (2005).

[7] N. B. Simeonova and W. K. Kegel, Phys. Rev. Lett. 93, 035701 (2004).

[8] E. Beerdsen et al., Phys. Rev. Lett. 95, 164505 (2005).

[9] R. Evans, in Fundamentals of Inhomogeneous Fluids, edited by D. Henderson (Dekker, New York, 1992), Chap. 3.

[10] U. Marini Bettolo Marconi and P. Tarazona, J. Chem. Phys. 110, 8032 (1999); J. Phys.: Condens. Matter 12, A413 (2000).

[11] Garnet Kin-Lic Chan and R. Finken, Phys. Rev. Lett. 94, 183001 (2005).

[12] J. Dzubiella and C. N. Likos, J. Phys.: Condens. Matter 15, L147 (2003); F. Penna, J. Dzubiella, and P. Tarazona, Phys. Rev. E 68, 061407 (2003); A. J. Archer, J. Phys.: Condens. Matter 17, 1405 (2005); M. Rex, H. Löwen, and C. N. Likos, Phys. Rev. E 72, 021404 (2005); M. Rex et al., Mol. Phys. 104, 527 (2006).

[13] C. P. Royall, J. Dzubiella, M. Schmidt, and A. van Blaaderen (unpublished).

[14] J. K. Percus, Phys. Rev. Lett. 8, 462 (1962).

[15] A. J. Archer and R. Evans, J. Chem. Phys. 121, 4246 (2004);
A. J. Archer and M. Rauscher, J. Phys. A 37, 9325 (2004).

[16] A. J. Archer, J. Phys.: Condens. Matter 18, 5617 (2006).

[17] B. Bagchi, Physica A 145, 273 (1987); K. Kawasaki, ibid. 208, 35 (1994).

[18] The ideal-gas free energy of one particle of species $s$ is (up to an additive constant) identical to the grand-canonical form in (2) and there is no self-interaction of species $s$ included in $F_{\text {ex }}$; see also J. A. White et al., Phys. Rev. Lett. 84, 1220 (2000) for an approach to canonical DFT.

[19] See, e.g., C. N. Likos, Phys. Rep. 348, 267 (2001).

[20] E. J. Saltzman and K. S. Schweizer, J. Chem. Phys. 125, 044509 (2006)

[21] T. V. Ramakrishnan and M. Yussouff, Phys. Rev. B 19, 2775 (1979).

[22] W. Kob et al., Phys. Rev. Lett. 79, 2827 (1997).

[23] D. Reguera and H. Reiss, J. Chem. Phys. 120, 2558 (2004).

[24] K. S. Schweizer and E. J. Saltzmann, J. Chem. Phys. 119, 1181 (2003); K. S. Schweizer, ibid. 123, 244501 (2005).

[25] W. van Megen and S. M. Underwood, Phys. Rev. Lett. 70, 2766 (1993).

[26] P. Tarazona, Phys. Rev. Lett. 84, 694 (2000).

[27] J. P. Stoessel and P. G. Wolynes, J. Chem. Phys. 80, 4502 (1984); Y. Singh, J. P. Stoessel, and P. G. Wolynes, Phys. Rev. Lett. 54, 1059 (1985); X. Xia and P. G. Wolynes, Proc. Natl. Acad. Sci. U.S.A. 97, 2990 (2000); Phys. Rev. Lett. 86, 5526 (2001); J. Phys. Chem. B 105, 6570 (2001); S. M. Bhattacharyya, B. Bagchi, and P. G. Wolynes, Phys. Rev. E 72, 031509 (2005).

[28] D. W. Oxtoby and R. Evans, J. Chem. Phys. 89, 7521 (1988). 